\title{
Covid-19: Two thirds of people contacted through tracing did not fully cooperate, pilot scheme finds
}

\author{
Elisabeth Mahase
}

The BMJ

A covid-19 contact tracing pilot scheme in Sheffield has found that two thirds of people contacted did not fully cooperate, and many of those worked in health and care settings.

The team behind the pilot have warned that "test and trace" schemes just launched in England and Scotland could be fraught with difficulties because of their centralised approach and their use of minimum wage employees at call centres. The team called for better messaging around the idea of civic duty to improve the chances of success.

The Sheffield Community Contact Tracers team was created by a group of retired doctors and public health experts and began the local pilot in April after they became frustrated by the lack of government action on contact tracing. ${ }^{1}$

In a report, ${ }^{2}$ the team described the inability or unwillingness to engage by people working in health and care settings as a "major challenge." They said that contact tracing may be resisted in workplaces, especially in the NHS and care homes, "where contact tracing systems, social distancing of staff, and a culture supportive of self-isolation frequently does not exist."

They also found that one in five of the initial patients responded to phone calls only after a physical message was hand delivered-something that the UK government may want to consider as it launches new NHS test and trace services.

Although the World Health Organization has repeatedly emphasised the importance of contact tracing, the UK abandoned the practice in March, arguing that resources were more effectively used elsewhere.

\section{Non-cooperation}

The Sheffield team initially recruited six volunteers, who received five hours' training and were assigned a medically qualified mentor to provide one-on-one support. A local general practice referred cases to the team. From these cases 13 people were enrolled, six of whom worked for the NHS or care services. The team followed up the cases until seven days after the date of first symptoms, and volunteers spent an average of 80 minutes on each case. Three referrals failed, one was inappropriate, one was dropped because of racist abuse, and one withdrew with no reason given.

The tracers identified 58 contacts, 19 of whom were named by the index cases. However, for the other 39 (two thirds) of the contacts, index cases were unable or unwilling to give their names and details. Of these 39 contacts, 29 worked for care provider agencies.
The team emphasised that contact tracing for covid-19 was different from existing tracing for diseases such as meningitis, where gains for the participant are immediate and clear. They also warned that the challenges faced by individual participants, including isolation in households and loss of income, might contribute to non-cooperation.

\section{Civic duty}

Joan Miller, retired public health doctor and a member of the Sheffield team, said, "Successful contact tracing takes meticulous hard work. In covid-19, this is also going to take tight liaison and cooperation between agencies. The voluntary and community sector have a very valuable role to play here alongside statutory bodies, because they reach communities others can't reach."

She added that the government's approach-using a call centre based national system-might be "too centralised and too remote."

Bing Jones, a member of the Sheffield pilot scheme and a former associate specialist in haematology, told The BMJ that the government's "call centre based, minimum wage based proposal is fraught with difficulties." He said that the government needed to communicate effectively to the public why isolating as a contact was important.

"We need to have very clear government messaging to beef up the notion of civic duty," he said. "There needs to be protocols and messaging which emphasises the need for people to do this thoroughly.

"There is no point in doing this half heartedly. It is very, very important that the government has clear messaging and information. We must encourage people and enable them to stay off work."

The Sheffield pilot has now expanded, recruiting and training 21 new volunteers. The team is also running a webinar on 29 May to help support other areas of the country in introducing their own local schemes. ${ }^{3}$

Mahase E. Covid-19: environmental health officers and retired doctors step up to fill contact tracing void. BMJ 2020;369:m1638. 10.1136/bmj.m1638 32332070

Community Contact Tracers. Sheffield Community Contact Tracers: a pilot to determine the feasibility of training community volunteers to undertake contact tracing for covid-19. the feasibility of training community volunteers to undertake contact tracing for covid-19. Pilot-Report-V2-Bing.pdf.

3 Community Contact Tracers. Sheffield Community Contact Tracers-evaluation findings webinar 29th May 2 pm. May 2020. https://docs.google.com/forms/d/e/

1FAlpQLSfSCSyzbReGach46W5quArIY0Wp709nh9HNWXyVG2f9yjmAHg/viewform. 
This article is made freely available for use in accordance with BMJ's website terms and conditions for the duration of the covid-19 pandemic or until otherwise determined by BMJ. You may use, download and print the article for any lawful, non-commercial purpose (including text and data mining) provided that all copyright notices and trade marks are retained.

https://bmj.com/coronavirus/usage 